\title{
Rotating Waves in a Model of Delayed Feedback Optical System with Diffraction
}

\author{
Stanislav BUDZINSKIY* \\ Faculty of Computational Mathematics and Cybernetics, Lomonosov Moscow State University, \\ Moscow 119991, Russia
}

\begin{abstract}
We study a delayed parabolic functional differential equation on a circle that is coupled with an initial value problem for the Schrodinger equation. Such equations arise as models of nonlinear optical systems with a timedelayed feedback loop, when diffusion of molecular excitation and diffraction are taken into account. The goal of this paper is to prove the existence of spatially inhomogeneous rotating-wave solutions bifurcating from homogeneous equilibria. We pass to a rotating coordinate system and seek an inhomogeneous solution to an ordinary functional differential equation. We find the solution in the form of a small parameter expansion and explicitly compute the first-order coefficients. We also provide examples of parameters that satisfy the constraints imposed throughout the analysis.
\end{abstract}

KEYWORDS: delay, parabolic functional differential equation, rotating wave, Kerr medium, diffraction

\section{Introduction}

Nonlinear optics is one of the areas where self-organization occurs [1]. A typical nonlinear optical system with rich spatio-temporal dynamics comprises a thin layer of nonlinear Kerr medium and a ring cavity (feedback loop). Phase modulation in the nonlinear Kerr slice within an aperture $Q \subset \mathbb{R}^{2}-$ real-valued function $u(\mathbf{r}, t)-$ is described by the following parabolic equation [2]:

$$
u_{t}(\mathbf{r}, t)+u(\mathbf{r}, t)-D \Delta u(\mathbf{r}, t)=\left|A_{F B}\right|^{2}, \quad \mathbf{r} \in Q, \quad t>0,
$$

augmented by boundary conditions on $\partial Q$ and by an initial condition. Here, $D>0$ is the diffusion coefficient; $A_{F B}$ is the complex amplitude of the light field after it has passed the feedback loop. Equation (1.1) includes local coupling caused by diffusion of molecular excitation in the nonlinear Kerr layer. Depending on the configuration of the feedback loop, expression for the complex amplitude $A_{F B}$ can bring nonlocal interactions into (1.1): time delay and/or spatial nonlocality (see $[3,4]$ ). Among the natural physical phenomena that can be taken into account in the mathematical model are interference of the input and feedback light fields [6], and free propagation diffraction in the feedback loop [5]. In the most general case, (1.1) is a delayed nonlinear partial functional differential equation [6].

The magnitude of nonlocalities together with the input light field intensity form an effective toolkit for controlling the dynamics of the system, which is crucial for applications (see [7,8]).

Whether Eq. (1.1) admits shape-preserving solutions is a matter of research. In [6], a periodic boundary value problem for Eq. (1.1) was shown to possess rotating-wave solutions, when time delay and rotation of spatial arguments are present. A similar equation was studied in case of Neumann boundary conditions and time delay [9]: only spatially homogeneous rotating waves were proved to exist.

In the present paper we study a mathematical model of an optical system with a thin ring aperture, time-delay device, interference, and diffraction. Our goal is to show that a one-dimensional periodic boundary value problem for Eq. (1.1) on a circle admits spatially inhomogeneous rotating-wave solutions bifurcating from spatially homogeneous equilibria. We prove the existence in a rotating coordinate system, seeking an inhomogeneous solution to an ordinary functional differential equation. We find the solution in the form of a small parameter expansion and explicitly compute the leading coefficients. We also provide examples of parameters that satisfy the constraints imposed throughout the analysis. 


\section{Boundary Value Problem}

\subsection{Problem statement}

We consider a periodic boundary value problem for a nonlinear parabolic functional differential equation on a circle:

$$
\begin{aligned}
u_{t}+u & =D u_{x x}+K\left|1+\gamma A\left(x, z_{0} ; e^{i u(t-T)}\right)\right|^{2}, \quad u=u(x, t), \quad x \in(0,2 \pi), \quad t>0, \\
\left.u\right|_{x=0} & =\left.u\right|_{x=2 \pi},\left.\quad u_{x}\right|_{x=0}=\left.u_{x}\right|_{x=2 \pi} .
\end{aligned}
$$

Equation (2.1) is an implementation of (1.1) for $A_{F B}=\sqrt{K}\left(1+\gamma A\left(x, z_{0} ; e^{i u(t-T)}\right)\right)$, where $K>0$ is the nonlinearity coefficient that is proportional to the input light field intensity, $0<\gamma<1$ is the interference visibility, $T>0$ is the time delay in the feedback loop, and $z_{0}>0$ is the free propagation distance of the light field. Here, $A\left(x, z ; A_{0}\right)$ denotes the solution to a periodic initial-boundary value problem for the linear Schrodinger equation that describes propagation of light waves in the paraxial approximation:

$$
\begin{aligned}
& A_{z}+i A_{x x}=0, \\
& \left.A\right|_{z=0}=A_{0}(x), \\
& \left.A\right|_{x=0}=\left.A\right|_{x=2 \pi},\left.\quad A_{x}\right|_{x=0}=\left.A_{x}\right|_{x=2 \pi} .
\end{aligned}
$$

For convenience, we shall introduce a linear operator $B$ that propagates its input over the distance $z_{0}$ according to (2.2):

$$
B:\left.H(\mathbb{C}) \ni A_{0}(x) \mapsto A\left(x, z ; A_{0}\right)\right|_{z=z_{0}} \in H(\mathbb{C}), \quad A_{0}(x) \in H_{2 \pi}^{2}(\mathbb{C}) .
$$

We now introduce the notation for functional spaces to be used in the sequel: $H$ is the Lebesgue space $L^{2}(0,2 \pi)$ of real-valued functions with the usual inner product; $H^{2}$ is the Sobolev space $W^{2,2}(0,2 \pi)$ of real-valued functions with the following inner product and norm

$$
\langle u, v\rangle_{H^{2}}=\int_{0}^{2 \pi}\left(u v+u^{\prime \prime} v^{\prime \prime}\right) d x, \quad\|u\|_{H^{2}}=\sqrt{\langle u, u\rangle_{H^{2}}}
$$

$H_{2 \pi}^{2}=\left\{u \in H^{2}: u(0)=u(2 \pi), u^{\prime}(0)=u^{\prime}(2 \pi)\right\}$ is a closed linear subspace of $H^{2}(\mathbb{C})$ consisting of periodic functions. Their complex-valued counterparts will be denoted by $H(\mathbb{C}), H^{2}(\mathbb{C})$, and $H_{2 \pi}^{2}(\mathbb{C})$, respectively; complex inner products are defined as $\langle u, v\rangle_{\mathbb{C}}=\langle u, \bar{v}\rangle$.

\section{Lemma 2.1.}

(1) The linear operator $B$ defined by (2.3) has a complete orthogonal set of eigenfunctions $\left\{e^{\text {inx }}\right\}_{n \in \mathbb{Z}}$. The corresponding eigenvalues are $\lambda_{n}(B)=e^{i n^{2} z_{0}}$.

(2) It is an isometry of $H(\mathbb{C})$.

(3) It is an isometry of $H_{2 \pi}^{2}(\mathbb{C})$ if treated as $B: H_{2 \pi}^{2}(\mathbb{C}) \longrightarrow H_{2 \pi}^{2}(\mathbb{C})$.

Proof.

(1) Let $A_{0}(x)=e^{i n x}$. The Fourier method applied to (2.2) readily gives $A(x, z)=e^{i n x} e^{i n^{2} z}$. The statement follows.

(2) Let $u \in H_{2 \pi}^{2}(\mathbb{C})$ and $e_{n}=\frac{1}{\sqrt{2 \pi}} e^{i n x}$. According to the Parseval's identity,

$$
\begin{aligned}
\|B u\|_{H(\mathbb{C})}^{2} & =\sum_{n=-\infty}^{+\infty}\left|\left\langle B u, e_{n}\right\rangle_{H(\mathbb{C})}\right|^{2}=\sum_{n=-\infty}^{+\infty}\left|\sum_{k=-\infty}^{+\infty}\left\langle u, e_{k}\right\rangle_{H(\mathbb{C})}\left\langle B e_{k}, e_{n}\right\rangle_{H(\mathbb{C})}\right|^{2} \\
& =\sum_{n=-\infty}^{+\infty}\left|e^{i n^{2} z_{0}}\left\langle u, e_{n}\right\rangle_{H(\mathbb{C})}\right|^{2}=\sum_{n=-\infty}^{+\infty}\left|\left\langle u, e_{n}\right\rangle_{H(\mathbb{C})}\right|^{2}=\|u\|_{H(\mathbb{C})}^{2}
\end{aligned}
$$

(3) Let $e_{n}=\frac{1}{\sqrt{2 \pi}\left(1+n^{4}\right)} e^{i n x}$. The proof is similar to (2).

Boundary value problem (2.1) admits spatially homogeneous equilibria $W(x, t) \equiv W$ that can be found as roots of

$$
F(W, K) \equiv W-K\left(1+2 \gamma \cos W+\gamma^{2}\right)=0 .
$$

Lemma 2.2. Suppose a pair $(\hat{W}, \hat{K})$ satisfies Eq. (2.4), and let the following nondegeneracy condition hold

$$
1+2 \hat{K} \gamma \sin \hat{W} \neq 0 \text {. }
$$

Then there exists a $\mu_{0}>0$ such that for $\mu \in\left(-\mu_{0}, \mu_{0}\right)$ a solution $(W(\mu), K(\mu))$ to Eq. (2.5) is defined and has the form

$$
K(\mu)=\hat{K}+\mu, \quad W(\mu)=\hat{W}+\hat{W}_{1} \mu+\hat{W}_{2} \mu^{2}+\ldots
$$

Proof. It follows from (2.5) that $\left.\frac{\partial F}{\partial W}\right|_{(\hat{W}, \hat{K})} \neq 0$. The statement is then a consequence of the analytic implicit function theorem.

We now bring boundary value problem (2.1) to the local form in the vicinity of a spatially homogeneous equilibrium 
$W(\mu)$ by setting $u(x, t)=W(\mu)+v(x, t)$ :

$$
\begin{aligned}
v_{t}+v & =D v_{x x}-W(\mu)+K(\mu)\left|1+\gamma e^{i W(\mu)} B e^{i v(t-T)}\right|^{2}, \\
\left.v\right|_{x=0} & =\left.v\right|_{x=2 \pi},\left.\quad v_{x}\right|_{x=0}=\left.v_{x}\right|_{x=2 \pi} .
\end{aligned}
$$

Extracting linear in $v$ terms we can obtain the following representation of (2.6):

$$
v_{t}+v=D v_{x x}+L(\mu) v(t-T)+F(v(t-T), \mu),
$$

where $L(\mu)$ and $F(\cdot, \mu)$ are operators of the form

$$
\begin{aligned}
L(\mu) w & =-2 \gamma K(\mu) \operatorname{Im}\left[\left(\gamma+e^{i W(\mu)}\right) B w\right], \\
F(w, \mu) & =\gamma K(\mu)\left\{2 \operatorname{Re}\left[\left(\gamma+e^{i W(\mu)}\right) B\left(e^{i w}-i w-1\right)\right]+\gamma\left|B\left(e^{i w}-1\right)\right|^{2}\right\} .
\end{aligned}
$$

Lemma 2.3. Let the assumptions of Lemma 2.2 hold. Then the operator $L(\mu)$ can be expanded in the vicinity of $\mu=0$ as

$$
L(\mu)=L_{0}+\mu L_{1}+\mu^{2} L_{2}(\mu)
$$

where

$$
\begin{aligned}
& L_{0} w=-2 \gamma \hat{K} \operatorname{Im}\left[\left(\gamma+e^{i \hat{W}}\right) B w\right], \\
& L_{1} w=-2 \gamma\left\{\hat{K} \operatorname{Re}\left[\hat{W}_{1} e^{i \hat{W}} B w\right]+\operatorname{Im}\left[\left(\gamma+e^{i \hat{W}}\right) B w\right]\right\}, \\
& \left\|L_{2}(\mu) w\right\|_{H} \leq C_{0}\|w\|_{H}, \quad \forall w \in H_{2 \pi}^{2} .
\end{aligned}
$$

Proof. Some algebra applied to (2.8) yields

$$
\begin{aligned}
L(\mu) w= & -2 \gamma(\hat{K}+\mu) \operatorname{Im}\left[\left(\gamma+e^{i W(\mu)}\right) B(w)\right] \\
= & \left\{e^{i W(\mu)}=e^{i \hat{W}}+\mu i \hat{W}_{1} e^{i \hat{W}}-e^{i \hat{W}}-\mu i \hat{W}_{1} e^{i \hat{W}}+e^{i W(\mu)}\right\} \\
= & -2 \gamma \hat{K} \operatorname{Im}\left[\left(\gamma+e^{i \hat{W}}\right) B(w)\right]-2 \gamma \mu\left\{\hat{K} \operatorname{Re}\left[\hat{W}_{1} e^{i \hat{W}} B(w)\right]+\operatorname{Im}\left[\left(\gamma+e^{i \hat{W}}\right) B(w)\right]\right\} \\
& -2 \gamma \mu^{2}\left\{\operatorname{Re}\left[\hat{W}_{1} e^{i \hat{W}} B(w)\right]+\hat{K} \operatorname{Im}\left[\left(-e^{i \hat{W}}-\mu i \hat{W}_{1} e^{i \hat{W}}+e^{i W(\mu)}\right) B(w)\right]\right\},
\end{aligned}
$$

which proves expansion (2.10). To show that $L_{2}(\mu)$ is bounded it suffices to use inequalities

$$
\left\|L_{2}(\mu) w\right\|_{H} \leq C\|B w\|_{H}, \quad w \in H_{2 \pi}^{2},
$$

where $C=2 \gamma\left\{\left|\hat{W}_{1}\right|+\hat{K}\left(2+\mu_{0}\left|\hat{W}_{1}\right|\right)\right\}$, and recall that $B$ is isometric according to Lemma 2.1.

Lemma 2.4. Let the assumptions of Lemma 2.2 hold. Then the operator $F(w, \mu)$ is analytic from a neighborhood of $(0,0) \in H_{2 \pi}^{2} \times \mathbb{R}$ into $H$. The operator $F$ and its Frechet derivatives $F_{w^{n} \mu^{m}}$ vanish at the origin $(0,0)$ for $n<2$.

Proof. The formal Taylor series expansion of $F(w, \mu)$ at the origin $(0,0)$ is

$$
F(w, \mu)=\sum_{n=0}^{\infty} \sum_{m=0}^{\infty}\left(\begin{array}{c}
n+m \\
n
\end{array}\right) \frac{1}{(n+m) !} F_{w^{n} \mu^{m}}(0,0) w^{n} \mu^{m} .
$$

Expanding $e^{i w}$ in its Taylor series and grouping the terms, one can show that $F_{w^{n}} \mu^{m}(0,0) w^{n} \mu^{m}=0$ for $n<2$. Hence, the expansion can be rewritten as

$$
F(w, \mu)=\sum_{n=2}^{\infty} \sum_{m=0}^{\infty} \frac{1}{n !} \frac{1}{m !} F_{w^{n} \mu^{m}}(0,0) w^{n} \mu^{m} .
$$

To prove the convergence of the Taylor series we derive an upper bound for the following expressions

$$
\frac{1}{k !(n-k) !}\left\|B w^{k} B w^{n-k}\right\|_{H(\mathbb{C})}, \quad w \in H_{2 \pi}^{2}, \quad n \geq 2, \quad k=0,1, \ldots, n .
$$

The embedding theorems [10] show that

$$
\begin{aligned}
& \|f g\|_{H^{2}(\mathbb{C})} \leq C_{0}\|f\|_{H^{2}(\mathbb{C})}\|g\|_{H^{2}(\mathbb{C})}, \quad \forall f, g \in H^{2}(\mathbb{C}), \\
& \left\|w^{m}\right\|_{H^{2}} \leq C_{0}^{m-1}\|w\|_{H^{2}}^{m}, \quad \forall w \in H^{2} .
\end{aligned}
$$

Therefore, recalling that $B$ is isometric, we can obtain the following bound

$$
\left\|B w^{k} B w^{n-k}\right\|_{H(\mathbb{C})} \leq\left\|B w^{k} B w^{n-k}\right\|_{H^{2}(\mathbb{C})} \leq C_{0}\left\|B w^{k}\right\|_{H^{2}(\mathbb{C})}\left\|B w^{n-k}\right\|_{H^{2}(\mathbb{C})}=C_{0}\left\|w^{k}\right\|_{H^{2}}\left\|w^{n-k}\right\|_{H^{2}} \leq C_{0}^{n-1}\|w\|_{H^{2}}^{n} .
$$

This leads to a majorant for the Taylor series term corresponding to $w^{n}$ :

$$
2 \gamma(\gamma+1)(\hat{K}+\mu) \frac{C_{0}^{n-1}\|w\|_{H^{2}}^{n}}{n !}+\gamma^{2}(\hat{K}+\mu) \sum_{k=0}^{n} \frac{C_{0}^{n-1}\|w\|_{H^{2}}^{n}}{k !(n-k) !}=(\hat{K}+\mu)\left(2 \gamma(\gamma+1)+2^{n} \gamma^{2}\right) \frac{C_{0}^{n-1}}{n !}\|w\|_{H^{2}}^{n},
$$


which means that the Taylor series expansion of $F(w, \mu)$ converges for any $w \in H_{2 \pi}^{2}$ and $|\mu|<\mu_{0}$ (as $W(\mu)$ is undefined elsewhere).

Direct computation gives a few Frechet derivatives:

$$
\begin{aligned}
F_{w w}(0,0) w^{2} & =-2 \gamma \hat{K}\left\{\operatorname{Re}\left[\left(\gamma+e^{i \hat{W}}\right) B w^{2}\right]-\gamma|B w|^{2}\right\}, \\
F_{w w w}(0,0) w^{3} & =2 \gamma \hat{K}\left\{\operatorname{Im}\left[\left(\gamma+e^{i \hat{W}}\right) B w^{3}\right]-3 \gamma \operatorname{Im}\left[\overline{B w} B w^{2}\right]\right\}, \\
F_{w w \mu}(0,0) w^{2} \mu & =2 \mu \gamma\left\{\hat{K} \operatorname{Im}\left[\hat{W}_{1} e^{i \hat{W}} B w^{2}\right]-\operatorname{Re}\left[\left(\gamma+e^{i \hat{W}}\right) B w^{2}\right]+\gamma|B w|^{2}\right\} .
\end{aligned}
$$

Hence, $F(w, \mu)$ can be represented as

$$
F(w, \mu)=\frac{1}{2} F_{w w}(0,0) w^{2}+\frac{1}{6} F_{w w w}(0,0) w^{3}+\frac{1}{2} F_{w w \mu}(0,0) w^{2} \mu+F_{4}(w, \mu),
$$

where $F_{4}(w, \mu)$ is analytic and contains terms $w^{n} \mu^{m}, n \geq 2, n+m>3$.

\subsection{Rotating coordinate system}

We approach the construction of rotating-wave solutions by passing to a rotating coordinate system. To this end, we introduce a family of rotation operators

$$
R_{\alpha} f(x)=f((x+\alpha) \bmod 2 \pi), \quad \alpha \in \mathbb{R}, \quad f \in H(\mathbb{C})
$$

and seek the solution $v(x, t)$ in the form

$$
v(x, t)=R_{-\Omega t} v(x), \quad \Omega \in \mathbb{R}, \quad v(x) \in H_{2 \pi}^{2} .
$$

\section{Lemma 2.5 .}

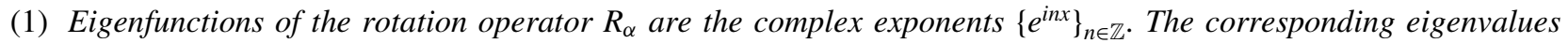
are $\lambda_{n}\left(R_{\alpha}\right)=e^{i n \alpha}$.

(2) The operators $B$ and $R_{\alpha}$ commute.

Proof. Direct computation proves the statement.

Ansatz (2.12) transforms boundary value problem (2.6) into

$$
\begin{aligned}
& D v^{\prime \prime}+\Omega v^{\prime}-v+L(\mu) R_{\Omega T} v+F\left(R_{\Omega T} v, \mu\right)=0, \\
& v(0)=v(2 \pi), \quad v^{\prime}(0)=v^{\prime}(2 \pi) .
\end{aligned}
$$

\section{Existence of Rotating Waves}

\subsection{Linearized operator}

To pursue the study of boundary value problem (2.13) we now turn to the linear part of the equation:

$$
A_{\Omega}: H \ni v \mapsto D v^{\prime \prime}+\Omega v^{\prime}-v+L_{0} R_{\Omega T} v \in H, \quad D\left(A_{\Omega}\right)=H_{2 \pi}^{2} .
$$

The $H$-adjoint operator of $A_{\Omega}$ is

$$
A_{\Omega}^{*}: H \ni u \mapsto D u^{\prime \prime}-\Omega u^{\prime}-u+R_{-\Omega T} L_{0}^{*} u \in H, \quad D\left(A_{\Omega}^{*}\right)=H_{2 \pi}^{2},
$$

with $L_{0}^{*}$ defined as

$$
L_{0}^{*} u=2 \gamma \hat{K} \operatorname{Im}\left[\left(\gamma+e^{-i \hat{W}}\right) B^{*} u\right]
$$

Here, the operator $B^{*}$ acts according to

$$
B^{*}:\left.E_{z_{0}}(x) \mapsto E\left(x, z ; E_{z_{0}}\right)\right|_{z=0}, \quad E_{z_{0}} \in H_{2 \pi}^{2}(\mathbb{C}),
$$

where $E\left(x, z ; E_{z_{0}}\right)$ is the solution to a periodic initial-boundary value problem for the adjoint Schrodinger equation

$$
\begin{aligned}
& E_{z}-i E_{x x}=0, \\
& \left.E\right|_{z=z_{0}}=E_{z_{0}}(x), \\
& \left.E\right|_{x=0}=\left.E\right|_{x=2 \pi},\left.\quad E_{x}\right|_{x=0}=\left.E_{x}\right|_{x=2 \pi} .
\end{aligned}
$$

Along with $A_{\Omega}$ we shall consider its complexification:

$$
A_{\Omega}^{\mathbb{C}} u=A_{\Omega} f+i A_{\Omega} g, \quad\left(A_{\Omega}^{\mathbb{C}}\right)^{*} u=\left(A_{\Omega}^{*}\right)^{\mathbb{C}} u=A_{\Omega}^{*} f+i A_{\Omega}^{*} g, \quad u=f+i g \in H_{2 \pi}^{2}(\mathbb{C}) .
$$

Lemma 3.1. The linear operators $A_{\Omega}^{\mathbb{C}}$ and $A_{\Omega}^{\mathbb{C} *}$ possess a complete orthogonal in $H(\mathbb{C})$ system of eigenfunctions $\left\{e^{i n x}\right\}_{n \in \mathbb{Z}}$. The corresponding eigenvalues are 


$$
\begin{aligned}
\lambda_{n}\left(A_{\Omega}^{\mathbb{C}}\right) & =-D n^{2}-1+i n \Omega-2 \gamma \hat{K} e^{i n \Omega T}\left\{\gamma \sin \left(n^{2} z_{0}\right)+\sin \left(\hat{W}+n^{2} z_{0}\right)\right\}, \\
\lambda_{n}\left(A_{\Omega}^{\mathbb{C} *}\right) & =-D n^{2}-1-i n \Omega-2 \gamma \hat{K} e^{-i n \Omega T}\left\{\gamma \sin \left(n^{2} z_{0}\right)+\sin \left(\hat{W}+n^{2} z_{0}\right)\right\} .
\end{aligned}
$$

Proof. According to Lemma 2.1 and Lemma 2.5,

$$
\begin{gathered}
B\left(R_{\Omega T} \cos n x\right)=e^{i n^{2} z_{0}} R_{\Omega T} \cos n x, \\
B\left(R_{\Omega T} \sin n x\right)=e^{i n^{2} z_{0}} R_{\Omega T} \sin n x .
\end{gathered}
$$

Hence,

$$
L_{0} R_{\Omega T} \cos n x+i L_{0} R_{\Omega T} \sin n x=-2 \gamma \hat{K} \operatorname{Im}\left[\left(\gamma+e^{i \hat{W}}\right) e^{i n^{2} z_{0}}\right] e^{i n \Omega T} e^{i n x}
$$

and, thus,

$$
A_{\Omega}^{\mathbb{C}} e^{i n x}=\left(-D n^{2}-1+i n \Omega-2 \gamma \hat{K} e^{i n \Omega T} \operatorname{Im}\left[\left(\gamma+e^{i \hat{W}}\right) e^{i n^{2} z 0}\right]\right) e^{i n x} .
$$

The case of the adjoint operator is similar.

Lemma 3.2. Let the assumptions of Lemma 2.2 hold. Then dimensions of $N\left(A_{\Omega}^{\mathbb{C}}\right)$ and $N\left(A_{\Omega}^{\mathbb{C} *}\right)$ are even.

Proof. According to (3.3), if $\lambda_{n}\left(A_{\Omega}^{\mathbb{C}}\right)=0$ then $\lambda_{-n}\left(A_{\Omega}^{\mathbb{C}}\right)=0$. So, only $n=0$ can make dimensions odd. But condition (2.5) states that

$$
\operatorname{Re} \lambda_{0}\left(A_{\Omega}^{\mathbb{C}}\right)=-(1+2 \gamma \hat{K} \sin \hat{W}) \neq 0
$$

Hence, dimensions are always even.

We now impose the main constraint on the parameters of the model.

Condition 3.3. Let $\Omega=\Omega_{*}$ be such that the system of equations

$$
\begin{aligned}
& 2 \gamma \hat{K} \cos (n \Omega T)\left\{\gamma \sin \left(n^{2} z_{0}\right)+\sin \left(\hat{W}+n^{2} z_{0}\right)\right\}=-D n^{2}-1, \\
& 2 \gamma \hat{K} \sin (n \Omega T)\left\{\gamma \sin \left(n^{2} z_{0}\right)+\sin \left(\hat{W}+n^{2} z_{0}\right)\right\}=\Omega n
\end{aligned}
$$

has exactly two solutions $n= \pm n_{*}, n_{*} \in \mathbb{N}$.

Lemma 3.4. Let Condition 3.3 hold. Then $N\left(A_{\Omega_{*}}^{\mathbb{C}}\right)=N\left(A_{\Omega_{*}}^{\mathbb{C} *}\right)=\operatorname{span}\left\{e^{i n_{*} x}, e^{-i n_{*} x}\right\}$.

Proof. According to Lemma 3.1, Condition 3.3 means that $\lambda_{n}\left(A_{\Omega_{*}}^{\mathbb{C}}\right)=0$ only for $n= \pm n_{*}$. The statement follows.

Lemma 3.5. Let Condition 3.3 hold. Then $R\left(A_{\Omega_{*}}\right)=N\left(A_{\Omega_{*}}^{*}\right)^{\perp}$.

Proof. (1) We start with the $R\left(A_{\Omega_{*}}\right) \subseteq N\left(A_{\Omega_{*}}^{*}\right)^{\perp}$ inclusion. Let $f \in R\left(A_{\Omega_{*}}\right)$; then a function $u$ exists in $D\left(A_{\Omega_{*}}\right)$ such that

$$
A_{\Omega_{*}} u=f
$$

and for all $g \in N\left(A_{\Omega_{*}}^{*}\right)$ the following equality holds:

$$
\langle f, g\rangle_{H}=\left\langle A_{\Omega_{*}} u, g\right\rangle_{H}=\left\langle u, A_{\Omega_{*}}^{*} g\right\rangle_{H}=0 .
$$

(2) To prove the inverse inclusion we consider an element $f \in N\left(A_{\Omega_{*}}^{*}\right)^{\perp}$. We expand $f$ into its Fourier series

$$
f=\sum_{n=-\infty}^{\infty} f_{n} e^{i n x}, \quad f_{-n}=\bar{f}_{n}, \quad f_{n_{*}}=f_{-n_{*}}=0
$$

and seek a solution $u \in D\left(A_{\Omega_{*}}\right)$ of (3.4) as a formal series

$$
u=\sum_{n=-\infty}^{\infty} u_{n} e^{i n x}
$$

From (3.4) we obtain equations

$$
u_{n} \lambda_{n}\left(A_{\Omega_{*}}\right)=f_{n}
$$

that are uniquely solvable for $n \neq \pm n_{*}$ :

$$
u_{n}=\frac{f_{n}}{-D n^{2}-1+i n \Omega-2 \gamma \hat{K} e^{i n \Omega T}\left\{\gamma \sin \left(n^{2} z_{0}\right)+\sin \left(\hat{W}+n^{2} z_{0}\right)\right\}}, \quad u_{-n}=\bar{u}_{n} .
$$

Setting $u_{n_{*}}=u_{-n_{*}}=0$, we check that the element $u$ defined by (3.5) indeed lies in $D\left(A_{\Omega_{*}}\right)$. Parseval's identity gives 


$$
\|u\|_{H^{2}}^{2}=\|u\|_{H}^{2}+\left\|u^{\prime \prime}\right\|_{H}^{2}=\frac{1}{2 \pi} \sum_{n=-\infty}^{\infty}\left(1+n^{4}\right)\left|u_{n}\right|^{2}=\frac{1}{2 \pi} \sum_{n=-\infty}^{\infty} c(n)\left|f_{n}\right|^{2} \leq C\|f\|_{H}^{2}
$$

with

$$
c(n)=\frac{1+n^{4}}{\left|-D n^{2}-1+i n \Omega-2 \gamma \hat{K} e^{i n \Omega T}\left\{\gamma \sin \left(n^{2} z_{0}\right)+\sin \left(\hat{W}+n^{2} z_{0}\right)\right\}\right|^{2}} \leq C .
$$

Hence, $u \in H^{2}$. To show periodicity it is sufficient to note that (3.5) and its termwise derivative converge uniformly on $[0,2 \pi]$.

Corollary 3.6. $R\left(A_{\Omega_{*}}\right)=N\left(A_{\Omega_{*}}\right)^{\perp}$.

Corollary 3.7. $R\left(A_{\Omega_{*}}\right)$ is closed in $H$.

Corollary 3.8. Let $\mathcal{P}: H_{2 \pi}^{2} \longrightarrow R\left(A_{\Omega_{*}}\right)$ be the projection operator. Then $A_{\Omega_{*}}: \mathcal{P} H_{2 \pi}^{2} \longrightarrow R\left(A_{\Omega_{*}}\right)$ is continuously invertible.

\subsection{Existence theorem}

In Section 2 we introduced two additional parameters that led us to boundary value problem (2.13): the small perturbation $\mu$ of the nonlinearity parameter $\hat{K}$ and the rotation speed $\Omega$ of the sought rotating-wave solution. These are now genuinely included in the following definition.

Definition 3.9. Let $S=(v, \Omega, \mu) \in H_{2 \pi}^{2} \times \mathbb{R} \times \mathbb{R}$. We call the triplet $S$ a solution to the boundary value problem (2.13) if $S$ turns (2.13) into identity.

It is evident that $S_{0}=\left(0, \Omega_{*}, 0\right)$ is a solution. We will be considering the nontrivial perturbations of $S_{0}$.

Condition 3.10. The following inequality holds:

$$
-2 \gamma n_{*}^{2}\left[\hat{K} \hat{W}_{1} \cos \left(\hat{W}+n_{*}^{2} z_{0}\right)+\sin \left(\hat{W}+n_{*}^{2} z_{0}\right)+\gamma \sin \left(n_{*}^{2} z_{0}\right)\right]\left[2 \gamma \hat{K} T\left\{\sin \left(\hat{W}+n_{*}^{2} z_{0}\right)+\gamma \sin \left(n_{*}^{2} z_{0}\right)\right\}-\cos \left(n_{*} \Omega_{*} T\right)\right] \neq 0 .
$$

Theorem 3.11. Let the assumptions of Lemma 2.2, Condition 3.3 and Condition 3.10 hold. Then an $\varepsilon_{0}>0$ exists such that for $\varepsilon \in\left(-\varepsilon_{0}, \varepsilon_{0}\right)$ a twice continuously differentiable with respect to $\varepsilon$ branch of solutions

$$
S_{\varepsilon}=\left(v(x ; \varepsilon), \Omega_{*}+\omega(\varepsilon), \mu(\varepsilon)\right) \in H_{2 \pi}^{2} \times \mathbb{R} \times \mathbb{R}
$$

is defined. Moreover, $S_{\varepsilon=0}=S_{0}$.

Proof. Consider a triplet $S=(v(\varepsilon), \Omega+\omega, \mu)$, where

$$
v(\varepsilon)=\varepsilon(\varphi+\xi), \quad \varphi=\frac{1}{\sqrt{\pi}} \cos \left(n_{*} x\right) \in N\left(A_{\Omega_{*}}\right), \quad \xi \in D\left(A_{\Omega_{*}}\right) \cap N\left(A_{\Omega_{*}}\right)^{\perp} .
$$

Plugging $S$ into (2.13) and dividing by $\varepsilon \neq 0$, we can get

$$
A_{\Omega_{*}} \xi+\omega\left(\varphi^{\prime}+\xi^{\prime}\right)+L(\mu) R_{\Omega T}(\varphi+\xi)-L_{0} R_{\Omega_{*} T}(\varphi+\xi)+\frac{1}{\varepsilon} F\left(R_{\Omega T} \varepsilon(\varphi+\xi), \mu\right)=0 .
$$

Subspaces $N\left(A_{\Omega_{*}}\right)=\operatorname{span}\left\{\varphi, \varphi^{\prime}\right\}$ and $R\left(A_{\Omega_{*}}\right)=N\left(A_{\Omega_{*}}\right)^{\perp}$ are invariant under the operators $d / d x, L(\mu), L_{0}\left(\right.$ since $\varphi$ and $\varphi^{\prime}$ are eigenfunctions of $B$ ). Therefore, we can project (3.7) onto the kernel and range:

$$
\begin{aligned}
& A_{\Omega_{*}} \xi+\omega \xi^{\prime}+L(\mu) R_{\Omega T} \xi-L_{0} R_{\Omega_{*} T} \xi+\frac{1}{\varepsilon} \mathcal{P} F\left(R_{\Omega T} v(\varepsilon), \mu\right)=0 \in R\left(A_{\Omega_{*}}\right), \\
& \omega\left\langle\phi^{\prime}, \phi\right\rangle_{H}+\left\langle L(\mu) R_{\Omega T} \phi, \phi\right\rangle_{H}-\left\langle L_{0} R_{\Omega_{*} T} \phi, \phi\right\rangle_{H}+\frac{1}{\varepsilon}\left\langle F\left(R_{\Omega T} v(\varepsilon), \mu\right), \phi\right\rangle_{H}=0 \in \mathbb{R}, \\
& \omega\left\langle\phi^{\prime}, \phi^{\prime}\right\rangle_{H}+\left\langle L(\mu) R_{\Omega T} \phi, \phi^{\prime}\right\rangle_{H}-\left\langle L_{0} R_{\Omega_{*} T} \phi, \phi^{\prime}\right\rangle_{H}+\frac{1}{\varepsilon}\left\langle F\left(R_{\Omega T} v(\varepsilon), \mu\right), \phi^{\prime}\right\rangle_{H}=0 \in \mathbb{R} .
\end{aligned}
$$

System (3.8) is equivalent to (3.7). It produces a nonlinear operator equation

$$
\mathcal{F}(\xi, \omega, \mu, \varepsilon)=0,
$$

where the operator

$$
\mathcal{F}: \mathcal{P} H_{2 \pi}^{2} \times \mathbb{R} \times \mathbb{R} \times \mathbb{R} \longrightarrow R\left(A_{\Omega_{*}}\right) \times \mathbb{R} \times \mathbb{R}
$$

is twice continuously Frechet differentiable in the vicinity of $(0,0,0,0)$.

We now seek an implicit function $(\xi, \omega, \mu)(\varepsilon)$ from (3.9). To this end, we compute the Frechet derivative of $\mathcal{F}$ at the origin $(0,0,0,0)$ with respect to $(\xi, \omega, \mu)$ :

$$
\nabla F=\left(\begin{array}{ccc}
A_{\Omega_{*}} & 0 & 0 \\
0 & \left\langle\phi^{\prime}, \phi\right\rangle+T\left\langle L_{0} R_{\Omega_{*} T} \phi^{\prime}, \phi\right\rangle & \left\langle L_{1} R_{\Omega_{*} T} \phi, \phi\right\rangle \\
0 & \left\langle\phi^{\prime}, \phi^{\prime}\right\rangle+T\left\langle L_{0} R_{\Omega_{*} T} \phi^{\prime}, \phi^{\prime}\right\rangle & \left\langle L_{1} R_{\Omega_{*} T} \phi, \phi^{\prime}\right\rangle
\end{array}\right) .
$$


According to Corollary 3.8, the linear operator $A_{\Omega_{*}}: \mathcal{P} H_{2 \pi}^{2} \longrightarrow R\left(A_{\Omega_{*}}\right)$ is continuously invertible. Thus, the linear operator

$$
\nabla F: \mathcal{P} H_{2 \pi}^{2} \times \mathbb{R} \times \mathbb{R} \longrightarrow\left(A_{\Omega_{*}}\right) \times \mathbb{R} \times \mathbb{R}
$$

is continuously invertible whenever the following matrix is nondegenerate:

$$
\nabla \mathcal{F}_{0}=\left(\begin{array}{cc}
\left\langle\phi^{\prime}, \phi\right\rangle+T\left\langle L_{0} R_{\Omega_{*} T} \phi^{\prime}, \phi\right\rangle & \left\langle L_{1} R_{\Omega_{*} T} \phi, \phi\right\rangle \\
\left\langle\phi^{\prime}, \phi^{\prime}\right\rangle+T\left\langle L_{0} R_{\Omega_{*} T} \phi^{\prime}, \phi^{\prime}\right\rangle & \left\langle L_{1} R_{\Omega_{*} T} \phi, \phi^{\prime}\right\rangle
\end{array}\right) .
$$

It follows from Condition 3.10 that $\operatorname{det} \nabla \mathcal{F}_{0} \neq 0$. The statement of the theorem is then a consequence of the twice continuously Frechet differentiable implicit operator theorem [11].

\subsection{Expansion coefficients}

In the proof of Theorem 3.11 we constructed a twice continuously differentiable triplet $(\xi, \omega, \mu)(\varepsilon)$. It can be expanded in powers of $\varepsilon$ as follows:

$$
\begin{aligned}
\xi(\varepsilon) & =\varepsilon \xi_{1}+\varepsilon^{2} \xi_{2}+\bar{o}\left(\varepsilon^{2}\right) \in \mathcal{P} H_{2 \pi}^{2}, \\
\omega(\varepsilon) & =\varepsilon \omega_{1}+\varepsilon^{2} \omega_{2}+\bar{o}\left(\varepsilon^{2}\right) \in \mathbb{R}, \\
\mu(\varepsilon) & =\varepsilon \mu_{1}+\varepsilon^{2} \mu_{2}+\bar{o}\left(\varepsilon^{2}\right) \in \mathbb{R} .
\end{aligned}
$$

We then plug expansion (3.10) into Eq. (3.7) to get the identity:

$$
\begin{aligned}
& A_{\Omega_{*}} \xi(\varepsilon)+\omega(\varepsilon) \frac{d}{d x}(\phi+\xi(\varepsilon))+L_{0}\left[R_{\Omega_{*} T+\omega(\varepsilon) T}(\phi+\xi(\varepsilon))-R_{\Omega_{*} T}(\phi+\xi(\varepsilon))\right]+\mu(\varepsilon) L_{1} R_{\Omega_{*} T+\omega(\varepsilon) T}(\phi+\xi(\varepsilon)) \\
& \quad+\mu^{2}(\varepsilon) L_{2}(\mu(\varepsilon)) R_{\Omega_{*} T+\omega(\varepsilon) T}(\phi+\xi(\varepsilon))+\frac{1}{\varepsilon} F\left(R_{\Omega_{*} T+\omega(\varepsilon) T} \varepsilon(\phi+\xi(\varepsilon)), \mu(\varepsilon)\right) \equiv 0 .
\end{aligned}
$$

From (3.10) we can deduce that

$$
R_{\Omega_{*} T+\omega(\varepsilon) T} y=R_{\Omega_{*} T} y+\omega(\varepsilon) T R_{\Omega_{*} T} y^{\prime}+\bar{o}(w(\varepsilon) T)=R_{\Omega_{*} T} y+\varepsilon \omega_{1} T R_{\Omega_{*} T} y^{\prime}+\bar{o}(\varepsilon) .
$$

The Taylor series (2.11) of the operator $F(w, \mu)$ together with (3.12) allow us to expand the nonlinear term of (3.11) in powers of $\varepsilon$ :

$$
F\left(R_{\Omega_{*} T+\omega(\varepsilon) T} \varepsilon(\phi+\xi(\varepsilon)), \mu(\varepsilon)\right)=\varepsilon^{2} F_{2}+\varepsilon^{3} F_{3}+\varepsilon^{4} F_{4}(\varepsilon),
$$

where

$$
\begin{aligned}
& F_{2}=\frac{1}{2} F_{w w}(0,0) R_{\Omega_{*} T}\left[\phi^{2}\right], \\
& F_{3}=F_{w w}(0,0) R_{\Omega_{*} T}\left[\phi \xi_{1}\right]+\omega_{1} T F_{w w}(0,0) R_{\Omega_{*} T}\left[\phi \phi^{\prime}\right]+\frac{1}{6} F_{w^{3}}(0,0) R_{\Omega_{*} T}\left[\phi^{3}\right]+\frac{1}{2} F_{w^{2} \mu}(0,0) R_{\Omega_{*} T}\left[\phi^{2} \mu_{1}\right], \\
& \left\|F_{4}(\varepsilon)\right\|_{H} \leq C(\Sigma), \quad \varepsilon \in \Sigma, \quad \forall \Sigma \Subset\left(-\varepsilon_{0}, \varepsilon_{0}\right) .
\end{aligned}
$$

We can now proceed with computing the first-order expansion coefficients $\xi_{1}, \omega_{1}$, and $\mu_{1}$. To this end, we collect the linear in $\varepsilon$ terms in (3.11):

$$
A_{\Omega_{*}} \xi_{1}=-\left[\omega_{1}\left(\phi^{\prime}+T L_{0} R_{\Omega_{*} T} \phi^{\prime}\right)+\mu_{1} L_{1} R_{\Omega_{*} T} \phi+F_{2}\right]
$$

By virtue of Corollary 3.6, Eq. (3.14) has a solution if and only if the right hand side is orthogonal to $N\left(A_{\Omega_{*}^{*}}^{*}\right)$; if a solution $\xi_{1} \in \mathcal{P} H_{2 \pi}^{2}$ exists, it is unique.

By definition, $F_{2} \in \operatorname{span}\left\{1, \cos \left(2 n_{*} x\right), \sin \left(2 n_{*} x\right)\right\}$ and is orthogonal to $N\left(A_{\Omega_{*}}^{*}\right)$ as a consequence. We, thus, get the following solvability condition:

$$
\begin{gathered}
\left\langle\omega_{1}\left(\phi^{\prime}+T L_{0} R_{\Omega_{*} T} \phi^{\prime}\right)+\mu_{1} L_{1} R_{\Omega_{*} T} \phi, \phi\right\rangle_{H}=0, \\
\left\langle\omega_{1}\left(\phi^{\prime}+T L_{0} R_{\Omega_{*} T} \phi^{\prime}\right)+\mu_{1} L_{1} R_{\Omega_{*} T} \phi, \phi^{\prime}\right\rangle_{H}=0 .
\end{gathered}
$$

This is a system of two linear equations; its matrix coincides with $\nabla \mathcal{F}_{0}$ from Theorem 3.11 , which is nondegenerate according to Condition 3.10. Therefore, Eq. (3.14) has a solution if and only if

$$
\omega_{1}=0, \quad \mu_{1}=0 .
$$

The solution $\xi_{1} \in \mathcal{P} H_{2 \pi}^{2}$ can be sought in the form

$$
\xi_{1}=c_{0}+c_{c} \cos \left(2 n_{*} x\right)+c_{s} \sin \left(2 n_{*} x\right) .
$$

This leads to the following expression: 


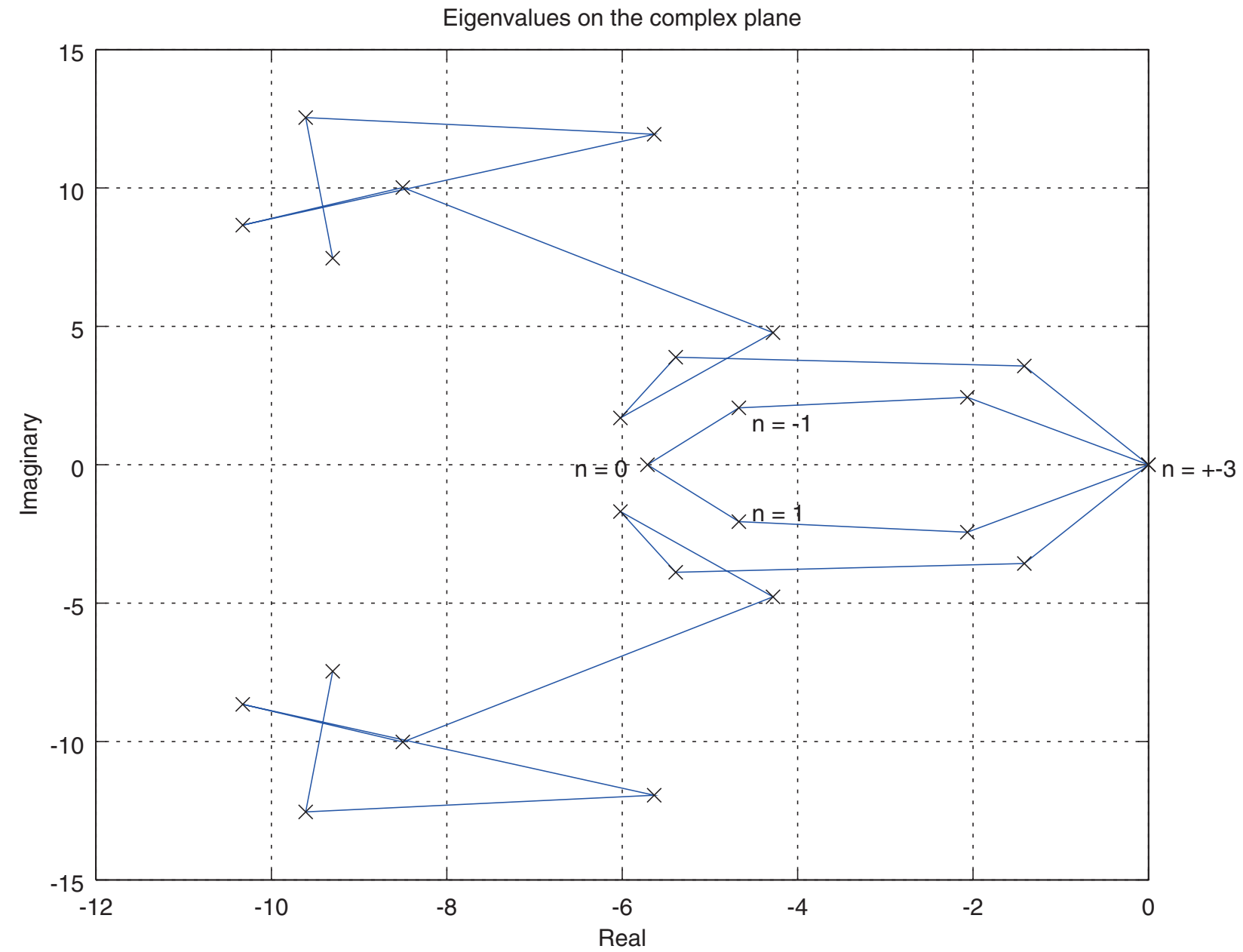

Fig. 1. Eigenvalues $\lambda_{n}\left(A_{\Omega_{*}}\right)$ plotted for $n \in[-12,12]$. The parameters are $n_{*}=3, \Omega_{*}=0.96191, \hat{W}=2.3562, \hat{K}=4.7124$, $\gamma=0.70711, D=0.074009, T=0.72577$, and $z_{0}=0.087266$.

$$
\xi_{1}=\frac{a_{0}}{\lambda_{0}\left(A_{\Omega_{*}}\right)}+\operatorname{Re}\left[\frac{a_{2}}{\lambda_{2 n_{*}}\left(A_{\Omega_{*}}\right)} R_{\Omega_{*} T} e^{i 2 n_{*} x}\right],
$$

which is well defined due to Condition 3.3. Here,

$$
a_{0}=\frac{1}{2 \pi} \gamma \hat{K} \cos \hat{W}, \quad a_{2}=\frac{1}{2 \pi} \gamma \hat{K}\left[\gamma\left(\cos \left(4 n_{*}^{2} z_{0}\right)-1\right)+\cos \left(\hat{W}+4 n_{*}^{2} z_{0}\right)\right] .
$$

To find second-order coefficients, we shall update expansion (3.12) of $R_{\Omega T}$ using (3.15):

$$
R_{\Omega_{*} T+\omega(\varepsilon) T} y=R_{\Omega_{*} T} y+\varepsilon^{2} \omega_{2} T R_{\Omega_{*} T} y^{\prime}+\bar{o}\left(\varepsilon^{2}\right) .
$$

We can now collect the $\varepsilon^{2}$ terms in (3.11):

$$
A_{\Omega_{*}} \xi_{2}=-\left[\omega_{2}\left(\phi^{\prime}+T L_{0} R_{\Omega_{*} T} \phi^{\prime}\right)+\mu_{2} L_{1} R_{\Omega_{*} T} \phi+F_{3}\right] .
$$

Equation (3.16) has a solution if and only if $\omega_{2}$ and $\mu_{2}$ satisfy the following system of linear equations:

$$
\begin{aligned}
\left\langle\omega_{2}\left(\phi^{\prime}+T L_{0} R_{\Omega_{*} T} \phi^{\prime}\right)+\mu_{2} L_{1} R_{\Omega_{*} T} \phi, \phi\right\rangle_{H} & =-\left\langle F_{3}, \phi\right\rangle_{H}, \\
\left\langle\omega_{2}\left(\phi^{\prime}+T L_{0} R_{\Omega_{*} T} \phi^{\prime}\right)+\mu_{2} L_{1} R_{\Omega_{*} T} \phi, \phi^{\prime}\right\rangle_{H} & =-\left\langle F_{3}, \phi^{\prime}\right\rangle_{H} .
\end{aligned}
$$

The matrix $\nabla \mathcal{F}_{0}$ is nondegenerate, so $\omega_{2}$ and $\mu_{2}$ can be found. To find the unique $\xi_{2}$, one can solve

$$
A_{\Omega_{*}} \xi_{2}=-\mathcal{P} F_{3} .
$$

\section{Examples of Parameters}

Below we present a few examples of parameters that satisfy the conditions of Lemma 2.2, Condition 3.3, and Condition 3.10. 


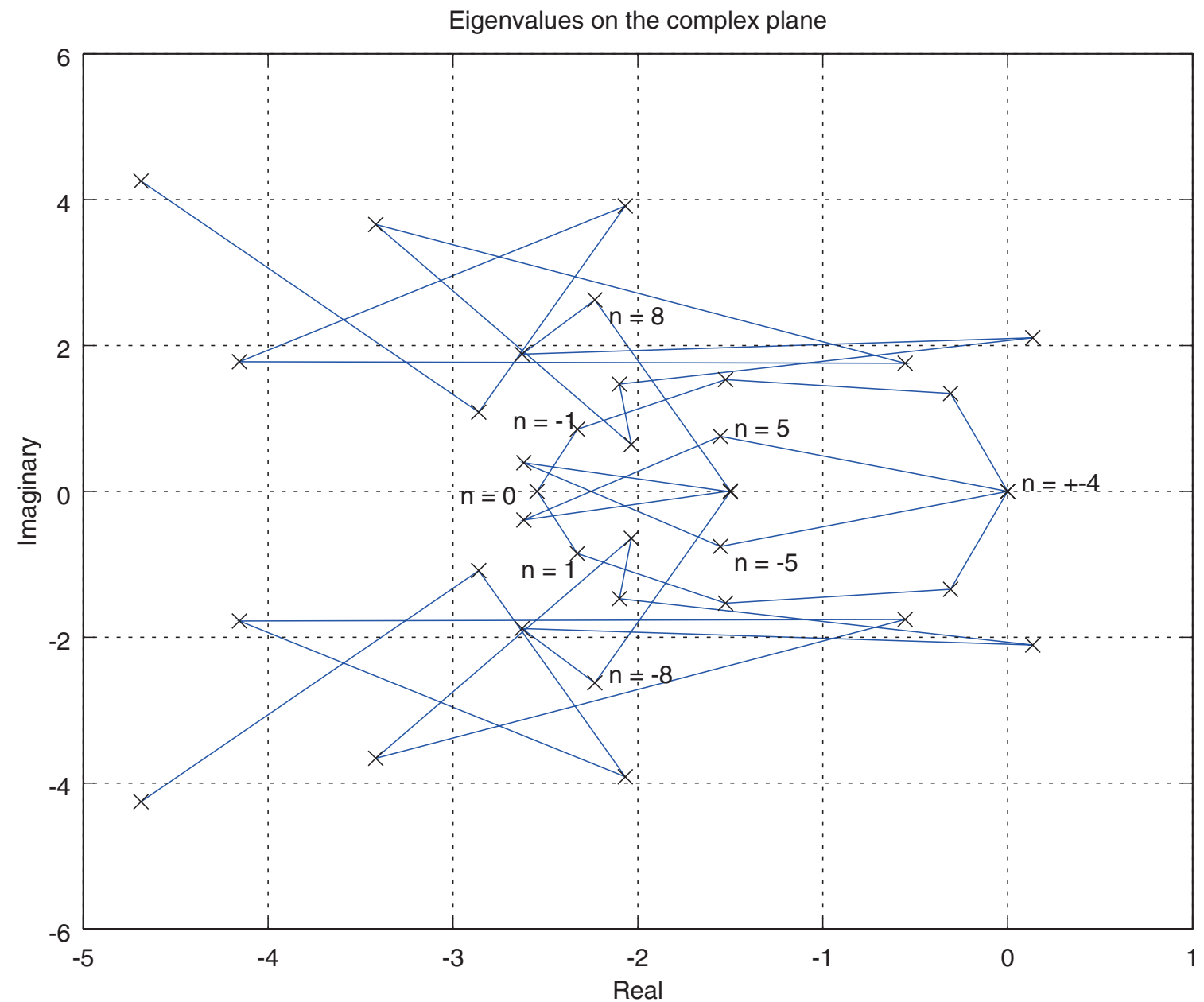

Fig. 2. Eigenvalues $\lambda_{n}\left(A_{\Omega_{*}}\right)$ plotted for $n \in[-18,18]$. The parameters are $n_{*}=4, \Omega_{*}=0.16094, \hat{W}=1.5708, \hat{K}=0.92703$, $\gamma=0.83333, D=0.007190, T=4.0667$, and $z_{0}=0.098175$.

- To show that $\pm n_{*}$ is the unique pair of roots in Condition 3.3 it is sufficient to check only for $|n| \leq 2 \gamma \hat{K}(\gamma+1.1) / \Omega_{*}$, since for bigger values we have $\left|\operatorname{Im} \lambda_{n}\right|>0.2 \gamma \hat{K}$.

- The expression in Condition 3.10 involves $\hat{W}_{1}$. It can be computed as $\hat{W}_{1}=\frac{1+2 \gamma \cos \hat{W}+\gamma^{2}}{1+2 \hat{K} \gamma \sin \hat{W}}$

\subsection{Example 1}

\begin{tabular}{|c|c|c|c|c|c|c|c|}
\hline$n_{*}$ & $\Omega_{*}$ & $\hat{W}$ & $\hat{K}$ & $\gamma$ & $D$ & $T$ & $z_{0}$ \\
\hline 3 & 0.96191 & 2.3562 & 4.7124 & 0.70711 & 0.074009 & 0.72577 & 0.087266 \\
\hline
\end{tabular}

- Expression in condition of Lemma 2.2 equals to $5.721 \neq 0$.

- Sufficient to check for $|n| \leq 2 \gamma \hat{K}(\gamma+1.1) / \Omega_{*}=11.896$. Figure 1 illustrates that Condition 3.3 is satisfied.

- Expression in Condition 3.10 equals to $-3.2513 \neq 0$.

\subsection{Example 2}

\begin{tabular}{|c|c|c|c|c|c|c|c|}
\hline$n_{*}$ & $\Omega_{*}$ & $\hat{W}$ & $\hat{K}$ & $\gamma$ & $D$ & $T$ & $z_{0}$ \\
\hline 4 & 0.16094 & 1.5708 & 0.92703 & 0.83333 & 0.007190 & 4.0667 & 0.098175 \\
\hline
\end{tabular}

- Expression in condition of Lemma 2.2 equals to $2.545 \neq 0$.

- Sufficient to check for $|n| \leq 2 \gamma \hat{K}(\gamma+1.1) / \Omega_{*}=17.696$. Figure 2 illustrates that Condition 3.3 is satisfied.

- Expression in Condition 3.10 equals to $-35.170 \neq 0$.

\subsection{Example 3}

\begin{tabular}{|c|c|c|c|c|c|c|c|}
\hline$n_{*}$ & $\Omega_{*}$ & $\hat{W}$ & $\hat{K}$ & $\gamma$ & $D$ & $T$ & $z_{0}$ \\
\hline 1 & 5.2477 & 7.864 & 6.882 & 0.3878 & 0.009241 & 0.335536 & 0.003142 \\
\hline
\end{tabular}




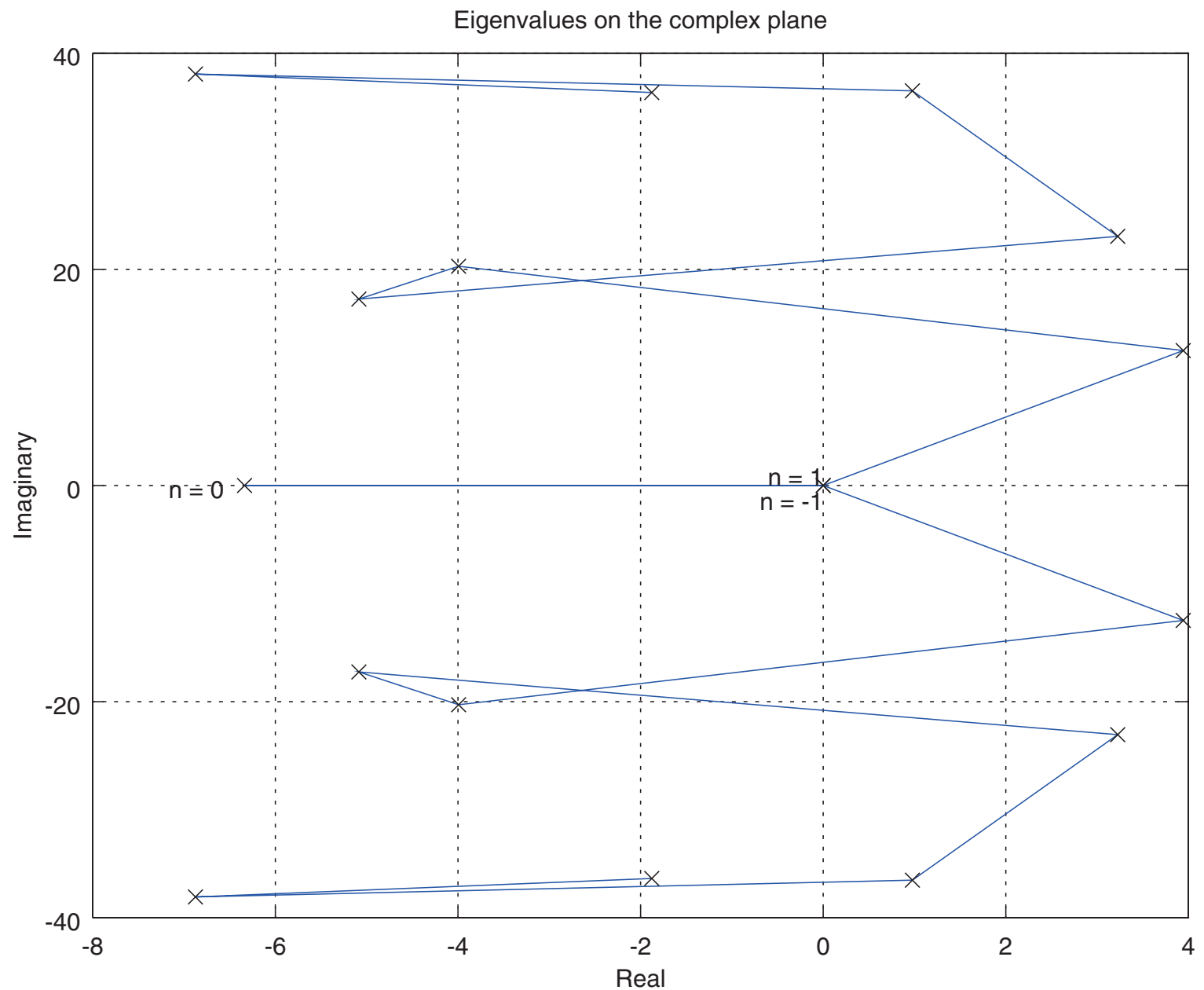

Fig. 3. Eigenvalues $\lambda_{n}\left(A_{\Omega_{*}}\right)$ plotted for $n \in[-8,8]$. The parameters are $n_{*}=1, \Omega_{*}=5.2477, \hat{W}=7.864, \hat{K}=6.882, \gamma=0.3878$, $D=0.009241, T=0.335536$, and $z_{0}=0.003142$.

- Expression in condition of Lemma 2.2 equals to $6.337 \neq 0$.

- Sufficient to check for $|n| \leq 2 \gamma \hat{K}(\gamma+1.1) / \Omega_{*}=1.422$. Figure 3 illustrates that Condition 3.3 is satisfied.

- Expression in Condition 3.10 equals to $-1.5138 \neq 0$.

\section{Acknowledgments}

The author is grateful to the organizers of GSIS Homogenization and Numerical Analysis International Summer School for giving an opportunity to share the present results with the other participants and to A.V. Razgulin for the statement of the problem and constant attention to the research.

\section{REFERENCES}

[1] Vorontsov, M. A., and Miller, W. B. (Eds.), Self-Organization in Optical Systems and Applications in Information Technology, Springer-Verlag, Berlin, Heidelberg (1995).

[2] Akhmanov, S. A., Vorontsov, M. A., Ivanov, V. Yu., Larichev, A. V., and Zheleznykh, N. I., "Controlling transverse-wave interactions in nonlinear optics: Generation and interaction of spatiotemporal structures," Journal of the Optical Society of America B, 9: 78-90 (1992).

[3] Vorontsov, M. A., and Iroshnikov, N. G., "Nonlinear dynamics of neuromorphic optical system with spatio-temporal interactions," Optical Memory and Neural Networks SPIE, 1621: 292-298 (1991).

[4] Chesnokov, S. S., Rybak, A. A., and Stadnichuk, V. I., "Time-delayed nonlinear optical systems: Temporal instability and cooperative chaotic dynamics," Proceedings of SPIE - The International Society for Optical Engineering, 4751: 493-498 (2002).

[5] Vorontsov, M. A., and Firth, W. J., "Pattern formation and competition in nonlinear optical systems with two-dimensional feedback," Physical Review A, 49(4): 2891-2906 (1994).

[6] Razgulin, A. V., and Romanenko, T. E., "Rotating waves in parabolic functional differential equations with rotation of spatial argument and time delay," Computational Mathematics and Mathematical Physics, 53(11): 1626-1643 (2013).

[7] Razgulin, A. V., "The problem of control of a two-dimensional transformation of spatial arguments in a parabolic functionaldifferential equation," Differential Equations, 42(8): 1140-1155 (2006). 
[8] Romanenko, T. E., and Razgulin, A. V., "Modeling of distortion suppression in a nonlinear optical system with a delayed feedback loop," Mathematical Models and Computer Simulations, 7(3): 259-270 (2015).

[9] Yoshida, K., "The Hopf bifurcation and its stability for semilinear diffusion equations with time delay arising in ecology," Hiroshima Mathematical Journal, 12(2): 321-348 (1982).

[10] Adams, R., and Fournier, J., Sobolev Spaces, Academic Press (2003).

[11] Zeidler, E., Applied Functional Analysis: Main Principles and Their Applications, Springer-Verlag (1995). 